\title{
Chiral capillary electrophoresis with UV-excited fluorescence detection for the enantioselective analysis of 9-fluorenylmethoxycarbonyl-derivatized amino acids
}

\author{
Amir Prior $^{1} \cdot$ Giulia Coliva $^{2} \cdot$ Gerhardus J. de Jong $^{2} \cdot$ Govert W. Somsen $^{1}$
}

Received: 21 February 2018 /Revised: 14 May 2018 / Accepted: 16 May 2018 / Published online: 29 May 2018

(C) The Author(s) 2018

\begin{abstract}
The potential of capillary electrophoresis (CE) with ultraviolet (UV)-excited fluorescence detection for sensitive chiral analysis of amino acids (AAs) was investigated. DL-AAs were derivatized with 9-fluorenylmethoxycarbonyl chloride (FMOC)-Cl to allow their fluorescence detection and enhance enantioseparation. Fluorescence detection was achieved employing optical fibers, leading UV excitation light $(<300 \mathrm{~nm})$ from a Xe-Hg lamp to the capillary window, and fluorescence emission to a spectrograph equipped with a charge-coupled device (CCD). Signal averaging over time and emission wavelength intervals was carried out to improve the signal-to-noise ratio of the FMOC-AAs. A background electrolyte (BGE) of $40 \mathrm{mM}$ sodium tetraborate (pH 9.5), containing $15 \%$ isopropanol $(v / v), 30 \mathrm{mM}$ sodium dodecyl sulfate (SDS), and $30 \mathrm{mM} \beta$-cyclodextrin $(\beta-\mathrm{CD})$, was found optimal for AA chemo- and enantioseparation. Enantioresolutions of 1.0 or higher were achieved for 16 proteinogenic DL-AAs. Limits of detection (LODs) were in the 10-100-nM range (injected concentration) for the D-AA enantiomers, except for FMOC-Dtryptophan $(536 \mathrm{nM})$ which showed intramolecular fluorescence quenching. Linearity $\left(R^{2}>0.997\right)$ and repeatability for peak height (relative standard deviations (RSDs) $<7.0 \% ; n=5$ ) and electrophoretic mobility (RSDs $<0.6 \% ; n=5$ ) of individual AA enantiomers were established for chiral analysis of DL-AA mixtures. The applicability of the method was investigated by the analysis of cerebrospinal fluid (CSF). Next to L-AAs, endogenous levels of D-glutamine and D-aspartic acid could be measured in CSF revealing enantiomeric ratios of 0.35 and $19.6 \%$, respectively. This indicates the method's potential for the analysis of low concentrations of D-AAs in presence of abundant L-AAs.
\end{abstract}

Keywords Amino acids - Chiral separation - Capillary electrophoresis - FMOC derivatization - Fluorescence detection . Cerebrospinal fluid

\section{Introduction}

Amino acids (AAs) play a major role in the physiology of organisms being building units of proteins but also essential in, e.g., metabolic processes, neurotransmission, and lipid transport. AAs are precursors for the synthesis of hormones and low-molecular-weight nitrogenous substances $[1,2]$. Most AAs in human life are chiral and predominantly occur

Govert W. Somsen

g.w.somsen@vu.nl

1 Division of BioAnalytical Chemistry, Amsterdam Institute for Molecules, Medicines and Systems, Vrije Universiteit Amsterdam, de Boelelaan 1085, 1081 HV Amsterdam, The Netherlands

2 Biomolecular Analysis, Utrecht University, Universiteitsweg 99, 3584 CG Utrecht, The Netherlands in their L-form. The presence of free D-AAs in the brain of humans and other mammals has been known for many years but for long time was thought to originate from bacteria [3] or formed by spontaneous racemization of L-AAs [4]. More recently, endogenous racemases were shown to be involved in D-AA synthesis $[5,6]$. Some D-AAs were found in relatively large quantities during the embryonic phase of brain development [7], localized to protoplasmic astrocytes, or closely distributed to NMDA receptors [8, 9], which suggests their function in signaling pathways, neurotransmission, and in brain biology [10]. D-AAs were found to have essential roles and abnormal D-AA levels and AA enantiomeric ratios in cerebrospinal fluid (CSF) may relate to neurodegeneration. Indeed, DAAs were found to be involved in the pathogenesis of psychiatric diseases and abnormal levels were associated with human disorders, such as schizophrenia and Alzheimer's disease [11-15]. For example, Samakashvili et al. [16] showed that 
chiral analysis of AAs in CSF might be useful for the early diagnosis and understanding of metabolism processes related to neurodegeneration and Alzheimer's disease. Clearly, analysis of D-AAs and determination of the D/L ratios can be of importance in clinical and pharmaceutical science but also in environmental and food analysis [17-20]. AA enantiomers of interest often are minor components of multi-component complex samples, such as tissues and biological fluids, requiring both chemo- and enantioselective separation with sensitive detection in order to allow their unambiguous assessment.

Chiral analysis of AAs can be performed by gas chromatography [21] and high-performance liquid chromatography $[22,23]$, requiring costly chiral stationary phases and/or relatively large amounts of (chiral) derivatization agents [24-27]. In addition, upon repeated analysis of biological samples, lifetimes of chiral columns may be limited. Capillary electrophoresis $(\mathrm{CE})$ has shown to be a versatile alternative tool for the enantioselective analysis of AAs requiring minute sample volumes and small amounts of solvents and chiral selector molecules. These selectors, such as (derivatized) cyclodextrins (CDs) [28, 29], vancomycin [30, 31], and 18-crown-6tetracarboxylic acid [32], can be simply added to the background electrolyte (BGE). A limitation of CE is the relatively low concentration sensitivity obtained with common ultraviolet (UV) absorbance detection, due to the small optical path length provided by the capillary internal diameter. Besides, only the aromatic AAs tryptophan, tyrosine, and phenylalanine show native UV absorbance. Therefore, AAs are often labeled with UV or visible light-absorbing agents and subsequently analyzed by CE with UV or fluorescence (Flu) detection [33-36]. The use of derivatization agents will not only improve detectability and sensitivity but also detection selectivity as only analytes with specific reactive groups will be derivatized and thus detected. Furthermore, derivatization of AAs may also enhance enantioseparation [37].

Chiral CE analysis of AAs with fluorescence detection has predominantly been done employing fluorescein isothiocyanate (FITC) [16, 33, 34, 38-40] but also, e.g., naphthalene2,3-dicarboxyaldehyde (NDA) [41], 4-fluoro-7-nitro-2,1,3benzoxadiazole (NBD-F) [42], 5-(4,6-dichloro-s-triazin-2ylamino) fluorescein (DTAF) [43], and 5-carboxyfluorescein succinimidyl ester (CFSE) [44] have been used for derivatization. These reagents, however, require relatively long derivatization times (30 min to several hours). More rapid derivatization (few minutes) can be achieved with 9fluorenylmethoxycarbonyl chloride (FMOC-Cl). Under alkaline conditions, FMOC reacts with primary and secondary amines and allows fast derivatization of all proteinogenic AAs [45]. Chiral CE-UV of FMOC-derivatized AAs has been described [45-48]. Although FMOC is fluorescent, and thus would allow more sensitive fluorescence detection, chiral CEFlu of FMOC-derivatized AAs has not been reported so far. This is most probably due to the fact that FMOC requires excitation in the UV region, which is not provided by conventional CE-Flu systems employing lasers with output in the visible region. Chan et al. [49] used a home-built laser-induced fluorescence (LIF) detector for the CE analysis of FMOC-AAs but did not perform chiral separations. In CE, UV-excited fluorescence detection of other compounds (e.g., vitamins, drugs, phenolic compounds, and proteins) has been achieved using a laser source (mostly $266 \mathrm{~nm}$ ) but also lampbased excitation using a Xe-Hg light source (for excitation in the range of $220-400 \mathrm{~nm}$ ) has been reported using a dedicated detection cell [50-55]. The latter showed up to 160 -fold sensitivity improvement as compared to UV absorbance detection, whereas sensitivity was comparable as obtained with LIF detection, while providing much higher flexibility in selection of UV excitation wavelengths.

In the present paper, we studied chiral CE-Flu of FMOCderivatized AAs employing lamp-based UV excitation. Using a CE-dedicated fluorescence setup [50-55], the light from a $\mathrm{Xe}-\mathrm{Hg}$ excitation lamp is led to the separation capillary by an optical fiber and focused onto the detection window. The analyte emission light is partly trapped within the fused silica capillary and guided along the capillary by total internal reflection. The fluorescence light is coupled out of the capillary by an optical cone and directed via a liquid light guide towards the detector, which was comprised of a spectrograph with a charge-coupled device (CCD) detector. Fluorescence excitation and emission parameters were studied in order to achieve optimal sensitivity of FMOC-AAs. Separation conditions were investigated and optimized for chiral and chemical resolution. Analytical aspects of the CE-Flu method, such as repeatability, linearity, and detection limits, were evaluated. The method's applicability was studied by the enantioselective analysis of AAs in CSF.

\section{Materials and methods}

\section{Chemicals}

All reagents were of analytical grade. FMOC-Cl, $\beta-\mathrm{CD}$, pentane, sodium tetraborate, sodium hydroxide, glycine, Dglutamic acid, D-histidine, D-threonine, L-alanine, L-arginine, L-asparagine, L-aspartic acid, L-cysteine, L-glutamic acid, Lglutamine, L-histidine, L-isoleucine, L-leucine, L-lysine, L-methionine, L-proline, L-serine, L-threonine, L-tryptophan, L-tyrosine and L-valine, DL-alanine, DL-arginine, DL-asparagine, DL-aspartic acid, DL-cysteine, DL-glutamic acid, DL-histidine, DL-isoleucine, DL-leucine, DL-lysine, DL-phenylalanine, DLproline, DL-serine, DL-tryptophan, and DL-valine were from Sigma-Aldrich (Steinheim, Germany). Isopropanol, DL-methionine, DL-tyrosine, sodium dodecyl sulfate, and acetonitrile were supplied by Fluka (Steinheim, Germany). Water was 
deionized and purified with a Milli-Q purification system (Millipore, Belford, NJ, USA).

The optimal BGE was $40 \mathrm{mM}$ sodium tetraborate (adjusted to $\mathrm{pH} 9.5$ with $1 \mathrm{M}$ sodium hydroxide) containing $15 \%(v / v)$ isopropanol, $30 \mathrm{mM}$ sodium dodecyl sulfate (SDS), and $30 \mathrm{mM} \beta-\mathrm{CD}$. The BGE was filtered prior to use through $0.45-\mu \mathrm{m}$ pore size disposable nylon filters from VWR (Amsterdam, The Netherlands). Stock solutions $(3 \mathrm{mM})$ of AAs were prepared in $0.2 \mathrm{M}$ sodium tetraborate ( $\mathrm{pH} 9.5$ ).

\section{Derivatization}

The $\mathrm{pH}$ of the CSF samples was adjusted by adding $10 \mu \mathrm{L}$ of $2 \mathrm{M}$ sodium hydroxide to $990 \mu \mathrm{L}$ CSF.

Derivatization of AAs with FMOC was carried out as described earlier [47]. Briefly, $500 \mu \mathrm{L}$ of $10 \mathrm{mM}$ FMOC in acetonitrile was added to $500 \mu \mathrm{L}$ sample (i.e., $\leq 3 \mathrm{mM} \mathrm{AA}$ in $0.2 \mathrm{M}$ sodium tetraborate buffer ( $\mathrm{pH} 9.5$ ) or $\mathrm{pH}$-adjusted CSF). This mixture was kept at room temperature for $2 \mathrm{~min}$ and then extracted with $1.5 \mathrm{~mL}$ pentane to remove excess of FMOC reagent. The aqueous phase was diluted ten times with water. The resulting solution was kept at $4{ }^{\circ} \mathrm{C}$ until injection.

\section{CE-Flu system}

CE experiments were carried out with a P/ACE MDQ CE instrument (Beckman Coulter, Brea, CA, USA). CE of AAs was performed using bare-fused silica capillaries (Polymicro Technologies, Phoenix, AZ, USA). The capillaries had an i.d. of $75 \mu \mathrm{m}$, an o.d. of $375 \mu \mathrm{m}$, and total/effective lengths of $72.2 / 55.3 \mathrm{~cm}$. The capillary temperature was set to $22{ }^{\circ} \mathrm{C}$. New bare-fused silica capillaries were rinsed with $1 \mathrm{M}$ sodium hydroxide for $10 \mathrm{~min}$ at $30 \mathrm{psi}$ and deionized water for $10 \mathrm{~min}$ at 30 psi. Between $\mathrm{CE}$ analyses, the capillaries were rinsed with BGE for 5 min at 30 psi. Overnight, the capillaries were stored in deionized water. Separations were performed in normal polarity mode with a separation voltage of $25 \mathrm{kV}$. Sample injection was performed hydrodynamically by applying $0.5 \mathrm{psi}$ for $13 \mathrm{~s}$, which corresponds to an injected volume of about $0.8 \%$ of the total capillary volume (BGE viscosity relatively to water $=1.93$ ). Data acquisition was performed using 32 Karat software (Beckman Coulter).

A previously described wavelength-resolved fluorescence (wrFlu) detector for CE was used, which was based on an Argos 250B fluorescence detection cell (Flux Instruments, Basel, Switzerland) [55] combined with a SR-163 spectrograph equipped with a CCD camera (Andor Technologies, Darmstadt, Germany) [56, 57]. The Argos system comprises a Xe-Hg lamp for excitation, excitation, and emission optical guides and filters and an optical cone detection cell. A capillary cartridge with an external detector adapter (Beckman Coulter) was used to guide the $\mathrm{CE}$ capillary from the inlet vial out of the $\mathrm{CE}$ instrument through the Argos fluorescence detector cell towards an external outlet vial containing the grounding electrode. The external detector adapter guarantees undisturbed capillary cooling by facilitating the CE instrument's liquid cooling. The emission light optical guide was connected to the spectrograph via a home-made fiber holder equipped with a back illuminated CCD chip of $256 \times 1024$ pixels with a pixel size of $26 \mu \mathrm{m}^{2}$ (Andor Technologies). The spectrograph comprised a grating of 600 lines $/ \mathrm{mm}$ blazed at $300 \mathrm{~nm}$ and a band-pass of $263 \mathrm{~nm}$. The CCD chip was cooled down to $-60{ }^{\circ} \mathrm{C}$. The spectrograph was wavelengthcalibrated daily using the reference spectral lines of an $\mathrm{Hg}$ pen-ray light source (L.O.T.-Oriel, Darmstadt, Germany). Typical detection settings used in CE-Flu experiments were slit width, $50 \mu \mathrm{m}$; exposure time, $3 \mathrm{~s}$; vertical shift speed, $16.25 \mu \mathrm{s}$; and horizontal read-out rate, $33 \mathrm{kHz}$. Acquired spectra were collected using the Full Vertical Binning mode and were background corrected. A 300-nm short-pass interference filter (Asahi Spectra USA Inc., Torrance, CA, USA) was used to select excitation light. Other tested excitation filters were a 260 ( \pm 10 )-nm band-pass filter (Asahi Spectra) and a 240400-nm broad-pass filter (Flux Instruments). Data acquisition analysis was performed using the software program Andor Solis (Andor Technologies).

\section{Fluorescence spectra}

Reference excitation and emission spectra of FMOC-AAs (10 $\mu \mathrm{M}$ in water) were recorded using an LS 50B fluorescence spectrometer (PerkinElmer, Waltham, MA) at room temperature using excitation and emission slit widths of 15 and $4 \mathrm{~nm}$, respectively, and a scan rate of $3 \mathrm{~nm} / \mathrm{s}$.

\section{Statement of human and animal rights}

No human or animal subjects were used in this study.

\section{Results and discussion}

\section{Fluorescence detection of FMOC-AAs}

Based on previous studies [29, 48, 58], a BGE of $40 \mathrm{mM}$ sodium tetraborate $(\mathrm{pH} 9.5)$ with $15 \%(v / v)$ isopropanol and $30 \mathrm{mM} \beta-\mathrm{CD}$ was selected as a starting condition to investigate the fluorescence detection of AA enantiomers. A test mixture of the DL-AAs alanine, aspartic acid, glutamic acid, leucine, methionine, and tryptophan was derivatized with FMOC-Cl. These AAs represent diverse chemical properties and exhibit different overall charge after derivatization. A bare-fused silica capillary with an i.d. of $75 \mu \mathrm{m}$ was used in order to maximize the optical path length for excitation, without inducing excessive CE current. Preliminary CE-UV experiments showed that under these conditions all test AAs were 
separated with an enantioresolutions ranging from 0.7 for alanine to 6.4 for glutamic acid.

The excitation and emission spectrum of FMOC-DL-phenylalanine was recorded using a conventional spectrofluorometer (Fig. 1A and B), clearly indicating that FMOC-AAs require excitation in the deep UV region for efficient fluorescence measurement. In order to achieve appropriate UV excitation, a CE-dedicated fluorescence detector equipped with a $\mathrm{Xe}-\mathrm{Hg}$ source was employed [55]. Previously, this system has shown useful for measuring native protein emission upon UV excitation [57]. The system comprises a spectrograph and CCD allowing on-line wavelength-resolved fluorescence (wrFlu) detection. The emission spectrum of FMOC-DL-phenylalanine recorded with wrFlu differed somewhat from the reference spectrum (Fig. 1B) showing a fluorescence maximum at $331 \mathrm{~nm}$. The difference in spectral shape and maximum wavelength is caused by a reduced transmittance for UV wavelengths below $315 \mathrm{~nm}$ of the detector optics (optical cone and emission light fiber) [57].

In order to achieve optimum detection of FMOC-AAs, excitation conditions were varied testing a 260-nm band-pass filter, a 300-nm cutoff short-pass filter, and a 240-400-nm broad-pass filter. A sample of FMOC-DL-aspartic acid $(1 \mu \mathrm{M})$ was repeatedly analyzed using the different excitation filters and the abovementioned BGE, which provided a resolution of 3.7 for the aspartic acid enantiomers. The signal-tonoise ratio $(S / N)$ obtained for each enantiomer at an emission wavelength of $331 \mathrm{~nm}$ was determined (Table 1). The lowest $S / N$ was observed using the $260-\mathrm{nm}$ band-pass excitation filter. Noise levels were relatively low with this filter, but absolute signal intensities were modest, as only a part of the excitation spectrum is employed to induce FMOC-aspartic acid fluorescence. Creating a broader band-pass by using the 300-nm cutoff short-pass filter or the 240-400-nm band-pass filter, an increase of $S / N$ was observed with respect to the 260$\mathrm{nm}$ filter. With the $240-400 \mathrm{~nm}$, much broader excitation indeed was achieved; however, it also significantly attenuated the overall excitation light intensity. Best $S / N$ was obtained by using only the 300-nm cutoff short-pass filter for excitation. Although noise levels significantly increased, absolute signal intensities were nine times higher as obtained with the $260-\mathrm{nm}$ band-pass filter, leading to most favorable detection of the FMOC-AA fluorescence.

The wrFlu detection provides the collection of a series of emission spectra over time. Using a detected emission wavelength of $331 \mathrm{~nm}$, only a fraction of the measured emission is used to construct an electropherogram. Integration of recorded emission intensities over a certain wavelength range for every measured point in time might be used to increase $S / N$ of the FMOC-AA signals. To evaluate this option, extracted electropherograms were constructed from the CE-Flu data obtained for FMOC-D-aspartic acid using the integrated signal of increasing wavelength intervals centered around $331 \mathrm{~nm}$ (Fig. 2). The $S / N$ grows steadily with increasing wavelength interval, until it levels off at a width of about $40 \mathrm{~nm}$. For wavelength intervals wider than $40 \mathrm{~nm}$, the integrated signal intensity does not significantly increase, while the integrated noise increases proportionally, yielding a loss in $S / N$. The gain obtained with wavelength interval integration is clearly illustrated by Fig. 3, showing the analysis of FMOC-DL-aspartic acid (1.25 $\mu \mathrm{M}$ for each enantiomer). Using signal integration, the $S / N$ increased with a factor of 12 with respect to single wavelength detection, leading to limits of detection (LODs) of less than $100 \mathrm{nM}$.

\section{Optimization of FMOC-AA separation}

In order to achieve enantioselective analysis of multiple AAs by CE-Flu, both chiral separation (i.e., enantioresolution) and mutual separation (i.e., chemoresolution) of the different AAs are required. Enhancement of AA separation can be attained by addition of SDS to the BGE, inducing micellar
Fig. 1 Excitation $(\mathbf{A}, \mathbf{C})$ and emission $(\mathbf{B}, \mathbf{D})$ spectra of FMOC-AAs $(10 \mu \mathrm{M})$ in water recorded with a standalone fluorescence spectrophotometer. $(\mathrm{A}+\mathrm{B})$ FMOC-phenylalanine. $(\mathrm{C}$ + D) FMOC-tryptophan Experimental conditions, see section "Materials and methods"

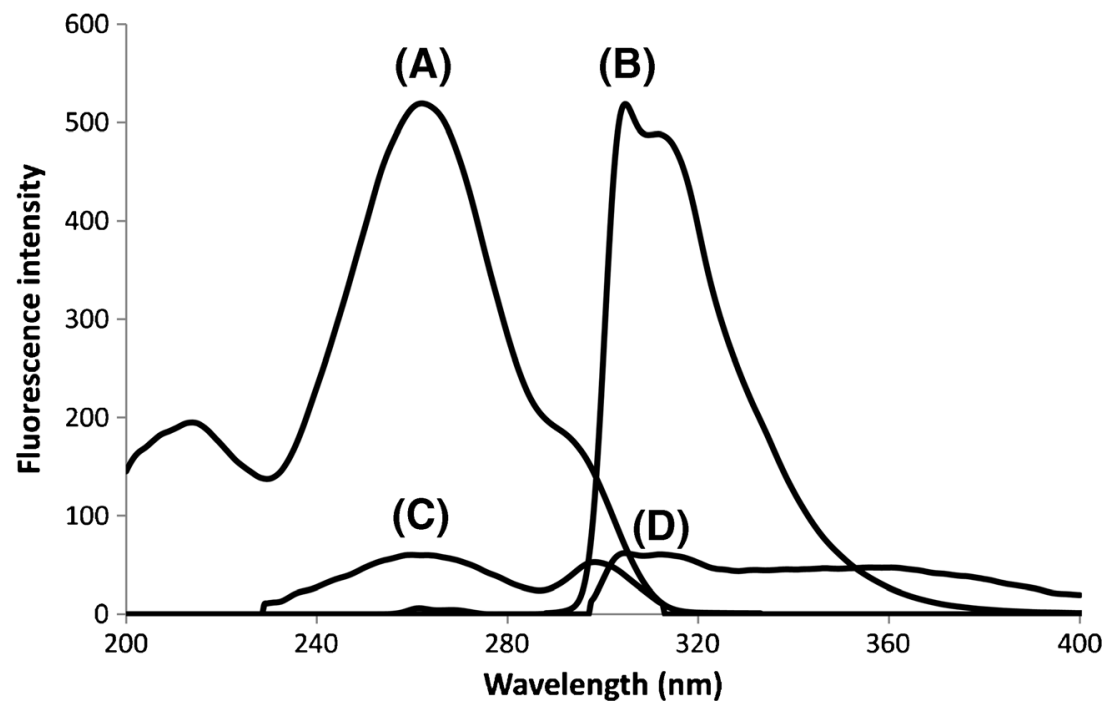


Table $1 \quad S / N$ s of FMOC-DL-aspartic acid $(1 \mu \mathrm{M}$ per enantiomer) obtained during CE-Flu using different excitation filters

\begin{tabular}{llll}
\hline Enantiomer & \multicolumn{2}{l}{ Excitation filter } \\
\cline { 2 - 4 } & $260 \mathrm{~nm}$ & $240-400 \mathrm{~nm}$ & $<300 \mathrm{~nm}$ \\
\hline D-Aspartic acid & 67.0 & 81.0 & 117.9 \\
L-Aspartic acid & 68.7 & 81.7 & 117.1 \\
\hline
\end{tabular}

Experimental conditions: emission wavelength, $331 \mathrm{~nm}$; for further conditions, see section "Materials and methods"

electrokinetic chromatography [29]. Therefore, a test mixture of 11 FMOC-DL-AAs (histidine, threonine, alanine, valine, methionine, isoleucine, glutamic acid, aspartic acid, leucine, phenylalanine, and tryptophan) was analyzed using BGEs of $40 \mathrm{mM}$ sodium tetraborate $(\mathrm{pH} 9.5)$ with $15 \%(v / v)$ isopropanol, $30 \mathrm{mM} \beta-\mathrm{CD}$, and 20,25 , or $30 \mathrm{mM}$ SDS. Raising the SDS concentration from 20 to $30 \mathrm{mM}$ resulted in longer analysis times (40, 47, and $83 \mathrm{~min}$, respectively) but also in an up to three times higher resolution of the AAs and an overall enhancement of enantioresolution (Fig. 4). Indeed, use of SDS in the BGE resulted in significantly increased enantioseparation of FMOC-AAs in comparison with $\mathrm{CE}$ employing a buffer with only $\beta-\mathrm{CD}$, as reported by us previously [58]. Highest enantioresolutions were observed at $30 \mathrm{mM}$ SDS for most tested FMOC-AAs, except for aspartic acid that was not significantly affected by the SDS concentration and glutamic acid that showed a decrease of enantioresolution with increasing SDS concentration. FMOC-aspartic acid and FMOC-glutamic acid are doubly negatively charged and most probably cannot partition into the SDS micelles due to electrostatic repulsion [46]. A BGE concentration of $30 \mathrm{mM}$ SDS was selected for further experiments.

The isopropanol content in the BGE was varied in the range of $13-17 \%$ in order to further fine-tune the separation of the 11 test FMOC-DL-AAs. Using 13\% isopropanol in the $\mathrm{BGE}$, the migration window of the tested FMOC-AAs was small, and as a result, many FMOC-AAs co-migrated. With 15 and $17 \%$ isopropanol in the BGE, the chemo- and enantioresolution improved significantly. With $17 \%$

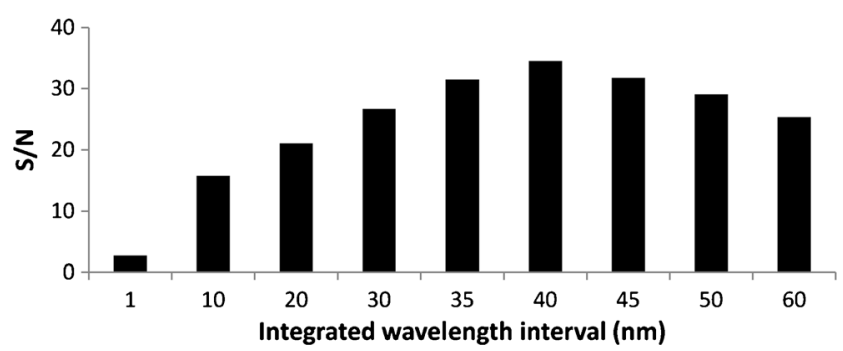

Fig. $2 S / N$ as function of the integrated wavelength interval obtained during CE-Flu of 1.25 $\mu \mathrm{M}$ FMOC-D-aspartic acid. For experimental conditions, see section "Materials and methods"

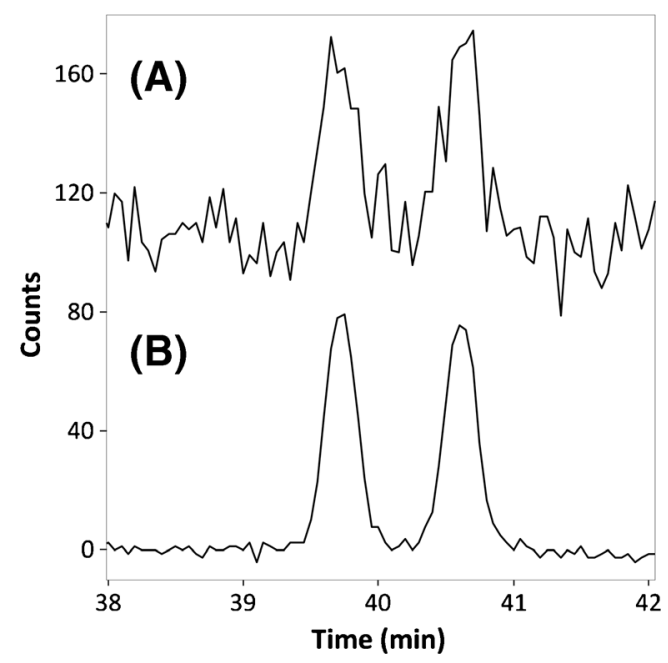

Fig. 3 Electropherograms obtained during chiral CE-Flu of FMOC-DLaspartic acid. (A) extracted electropherogram at an emission wavelength of $331 \mathrm{~nm}$ and (B) extracted electropherogram using emission signal averaging over 40-nm interval centered around $331 \mathrm{~nm}$. Injected concentrations, $1.25 \mu \mathrm{M}$ of each enantiomer; D-enantiomer migrates before L-enantiomer. For further experimental conditions, see section "Materials and methods"

isopropanol, 19 out of the 22 FMOC-AA enantiomers were mutually resolved, whereas with $15 \%$ isopropanol almost full separation was achieved with only L-aspartic acid and D-leucine co-migrating. The optimum BGE was $40 \mathrm{mM}$ sodium tetraborate ( $\mathrm{pH} 9.5)$ with $15 \%(v / v)$ isopropanol, $30 \mathrm{mM} \beta$ $\mathrm{CD}$, and $30 \mathrm{mM}$ SDS.

Finally, the effect of the capillary thermostating temperature $\left(15-23^{\circ} \mathrm{C}\right)$ on the analysis time and resolution was studied. Increasing the capillary temperature overall resulted in shorter migration times. For instance, at $15^{\circ} \mathrm{C}$, the migration time of DL-leucine was about $59 \mathrm{~min}$, whereas at $23^{\circ} \mathrm{C}$, the enantiomers were detected after $44 \mathrm{~min}$. Although for most AAs the enantioresolution slightly decreased with raising capillary temperatures, the chemoresolution of the AAs increased. As a compromise between analysis time, chemoresolution, and enantioresolution, a capillary temperature of $22{ }^{\circ} \mathrm{C}$ was selected.

Figure 5 shows the CE-Flu analysis of the 11 test DLAAs derivatized with FMOC using the optimized method. All analyzed FMOC-AAs show enantioresolution $(1.0-8.8)$ and are almost fully separated mutually. The system peak from the unreacted FMOC reagent is not interfering with the FMOC-AAs. Tryptophan and phenylalanine showed relatively long migration times, most probably due to their relatively high hydrophobicity and, therefore, strong interaction with the SDS micelles.

\section{Analytical performance}

For the optimized CE-Flu method, precision of migration time and electrophoretic mobility were assessed by analyzing the 
Fig. 4 Effect of SDS

concentration in the BGE on the FMOC-AA enantiomeric resolution. BGE, $40 \mathrm{mM}$ sodium tetraborate ( $\mathrm{pH}$ 9.5) containing $15 \%$ isopropanol, $30 \mathrm{mM} \beta-\mathrm{CD}$ and SDS. For further

experimental conditions, see section "Materials and methods". Asterisk: At $20 \mathrm{mM}$ SDS in the $\mathrm{BGE}$, the histidine enantiomers co-migrated with unreacted FMOC and could not be observed. Double asterisk: At $20 \mathrm{mM}$ SDS in the BGE, the alanine enantiomers were not separated
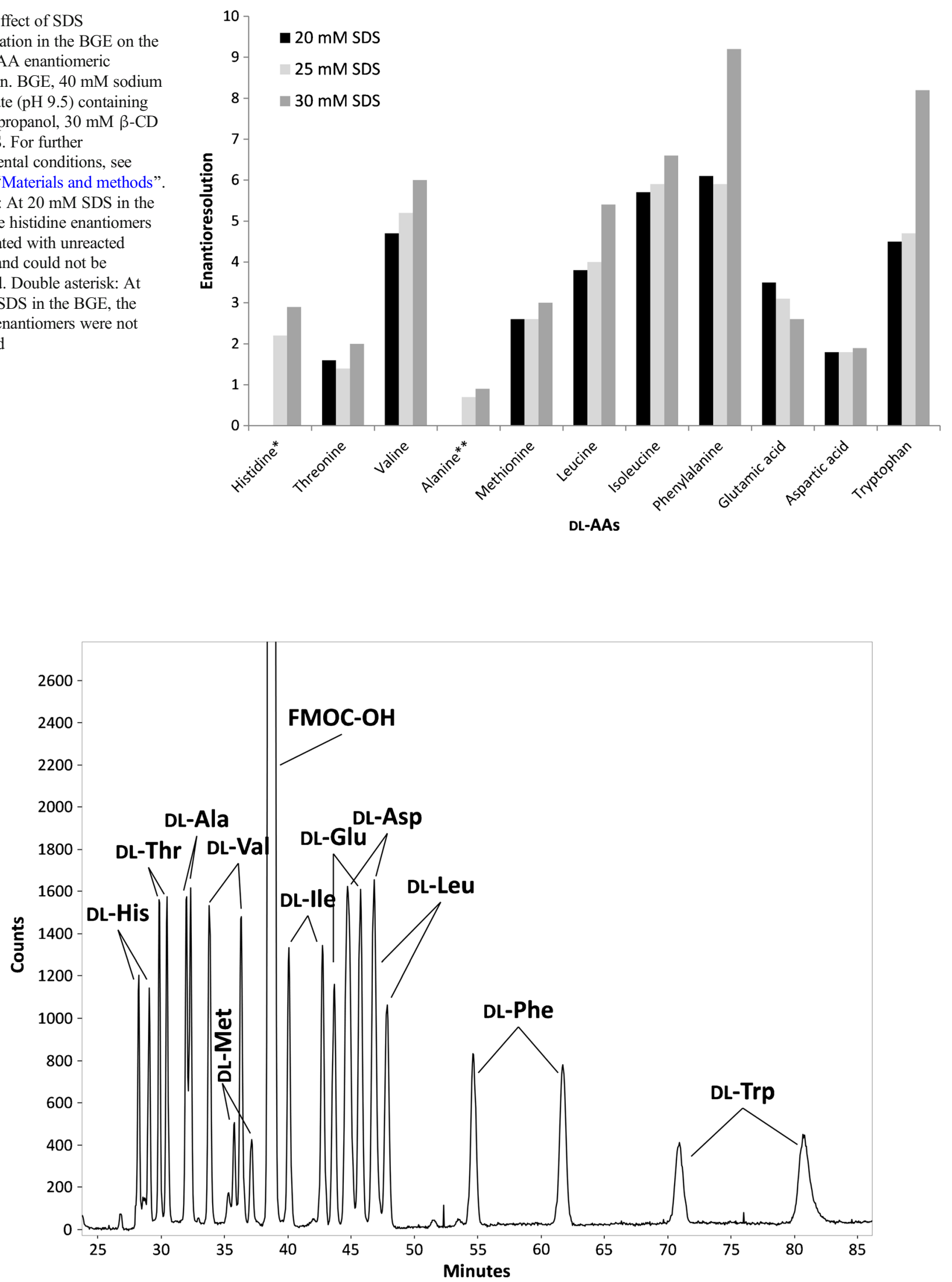

Fig. 5 Electropherogram obtained during chiral CE-Flu of a mixture of 11 DL-AAs. For all FMOC-AAs, the D-form migrates before the L-form. Injected concentrations, $500 \mathrm{nM}$ for each enantiomer, except for tryptophan, $5000 \mathrm{nM}$. For further experimental conditions, see section "Materials and methods" 
11 DL-AAs mixture in five consecutive runs, yielding relative standard deviations (RSDs) in the range of 1.6-5.8 and 0.1$0.6 \%$, respectively. RSDs for peak height were in the range of 1.6-7.0\%. Method linearity was assessed by derivatizing mixtures of DL-threonine and DL-leucine, which represent a fast and a slow migrating AA, of different concentration (75$3700 \mathrm{nM}$ for each enantiomer). Good linearity was observed for the enantiomers of both AAs with coefficients of determination $\left(R^{2}\right)$ above 0.997 .

The chiral performance and sensitivity of the optimized CE-Flu method were evaluated for 19 chiral proteinogenic AAs and glycine (Table 2). Tyrosine, lysine, and cysteine were not detected within 90 min of analysis. Lysine and cysteine-which carry two FMOC moieties after derivatization [29] - and tyrosine are quite hydrophobic and show high affinity for the SDS micelles, yielding very low mobility. Extending the analysis time revealed the enantioseparation of these AAs with migration times of up to $3.5 \mathrm{~h}$. The other FMOC-AAs were successfully enantioseparated, showing resolution of 1.5 or higher, except for alanine (resolution, 1.0). The LODs (injected concentration yielding a $S / N$ of 3 ) were in the range of $14-98 \mathrm{nM}(2-15 \mathrm{ng} / \mathrm{mL})$, except for the tryptophan enantiomers, which exhibited a LOD of $536 \mathrm{nM}$ $(110 \mathrm{ng} / \mathrm{mL})$. The intensity of the FMOC-tryptophan excitation and emission spectra (Fig. 1C, D) indeed was lower than the intensity observed for the other FMOC-AAs

Table 2 Enantiomer resolution and LODs (nM; ng/mL) obtained for 17 proteinogenic AAs using chiral CE-Flu

\begin{tabular}{llll}
\hline Amino acid $^{\mathrm{a}}$ & Enantioresolution & LOD $(\mathrm{nM})^{\mathrm{b}}$ & LOD $(\mathrm{ng} / \mathrm{mL})^{\mathrm{b}}$ \\
\hline Alanine & 1.0 & 22 & 1.9 \\
Valine & 7.4 & 19 & 2.2 \\
Methionine & 3.9 & 39 & 5.8 \\
Threonine & 2.2 & 21 & 2.5 \\
Histidine & 3.4 & 98 & 15.2 \\
Isoleucine & 5.4 & 52 & 6.8 \\
Glutamic acid & 3.8 & 19 & 2.8 \\
Aspartic acid & 1.9 & 27 & 3.6 \\
Leucine & 5.4 & 37 & 4.8 \\
Phenylalanine & 8.8 & 28 & 4.6 \\
Tryptophan & 7.1 & 536 & 109.4 \\
Glycine & - & 27 & 2.0 \\
Proline & 1.5 & 38 & 4.3 \\
Serine & 2.1 & 16 & 1.7 \\
Asparagine & 2.1 & 15 & 1.9 \\
Glutamine & 1.7 & 14 & 2.0 \\
Arginine & 3.4 & 36 & 6.2 \\
\hline
\end{tabular}

${ }^{a}$ Injected concentration, $500 \mathrm{nM}$ per enantiomer (except tryptophan, $5000 \mathrm{nM}$ )

${ }^{\mathrm{b}}$ Concentration yielding a $S / N$ of 3 as calculated for the D-enantiomer
(Fig. 1A, B) for the same concentration. The lower fluorescence yield of FMOC-tryptophan is due to intramolecular quenching of the FMOC emission by the indole moiety of tryptophan [59]. On average, the obtained LODs encompass an improvement of the sensitivity of two orders of magnitude when compared with chiral CE-UV methods for AAs using FMOC derivatization [45-48]. These studies reported LODs in the micromolar range.

\section{Application to CSF}

The feasibility of the developed chiral CE-Flu method for the detection of D-AAs in biofluids was investigated by the analysis of CSF. CSF was spiked with 13 DL-AAs at levels corresponding to a concentration of $250 \mathrm{nM}$ in CSF for each enantiomer (except for tryptophan, $2500 \mathrm{nM}$ ) and analyzed by CEFlu (Fig. 6A). Assignment of the peaks was performed by spiking CSF with individual FMOC-DL-AAs. All the tested FMOC-AAs could be detected in the CSF and each was enantioseparated. Nevertheless, for CSF, no chemoresolution of histidine and glutamine and of threonine and serine was achieved and the L-enantiomers of glutamic acid and aspartic acid co-migrated. Analysis of blank CSF (Fig. 6B) showed the natural presence of the L-enantiomers of glutamine, histidine, serine, threonine, alanine, valine, methionine, isoleucine, glutamic acid, aspartic acid, phenylalanine, and tryptophan. More importantly, the sensitivity of the CE-Flu method allowed direct detection of D-aspartic acid in the blank CSF (peak at $48 \mathrm{~min}$ in Fig. 6B). In addition, the small peak at 26 min (Fig. 6B) was assigned to D-glutamine as D-histidine is not expected to be present in CSF [11]. From the measured peak areas, the $\mathrm{D} / \mathrm{L}$-enantiomeric ratio of aspartic acid in CSF was calculated to be $19.6 \%$. For glutamine, the D/L-enantiomeric ratio was estimated to be $0.35 \%$. These enantiomeric ratios are within reported ranges for aspartic acid (18-25\%) and glutamine $(0.1-1.0 \%)$ in CSF $[60,61]$. Based on the peak areas and the spiked concentrations, endogenous CSF levels for D-aspartic acid and D-glutamine of 1365 and $565 \mathrm{nM}$ (182 and $82 \mathrm{ng} / \mathrm{mL}$, respectively) were estimated, which is within reported ranges for these two D-AAs [61, 62]. In order to appreciate the LOD of the CE-Flu method for D-AA analysis in CSF, DL-leucine was selected as this AA was not present in the blank CSF analyzed. From the peak area obtained for the spiked CSF, the LOD for D-leucine in CSF was determined to be $1050 \mathrm{nM}(138 \mathrm{ng} / \mathrm{mL})$ which corresponds to an injected concentration of $52 \mathrm{nM}(6.8 \mathrm{ng} / \mathrm{mL})$ taking the dilution from the sample pretreatment into account. This value is similar to the LOD obtained for leucine in aqueous solution (Table 2), indicating minor effects of the CSF matrix on the analysis of the AAs. Overall, these results indicate the potential of the CE-Flu method to detect D-AAs next to their L-AA enantiomers in a biofluid. 
Fig. 6 Electropherograms obtained during chiral CE-Flu of (A) CSF spiked with 13 DL-AAs, and (B) blank CSF. For (A)

$5.00 \mu \mathrm{M}$ per enantiomer was spiked into the CSF, except for tryptophan $(50.0 \mu \mathrm{M})$, which

corresponds to injected concentrations of 250 and $2500 \mathrm{nM}$, respectively. For further experimental conditions, see section "Materials and methods"
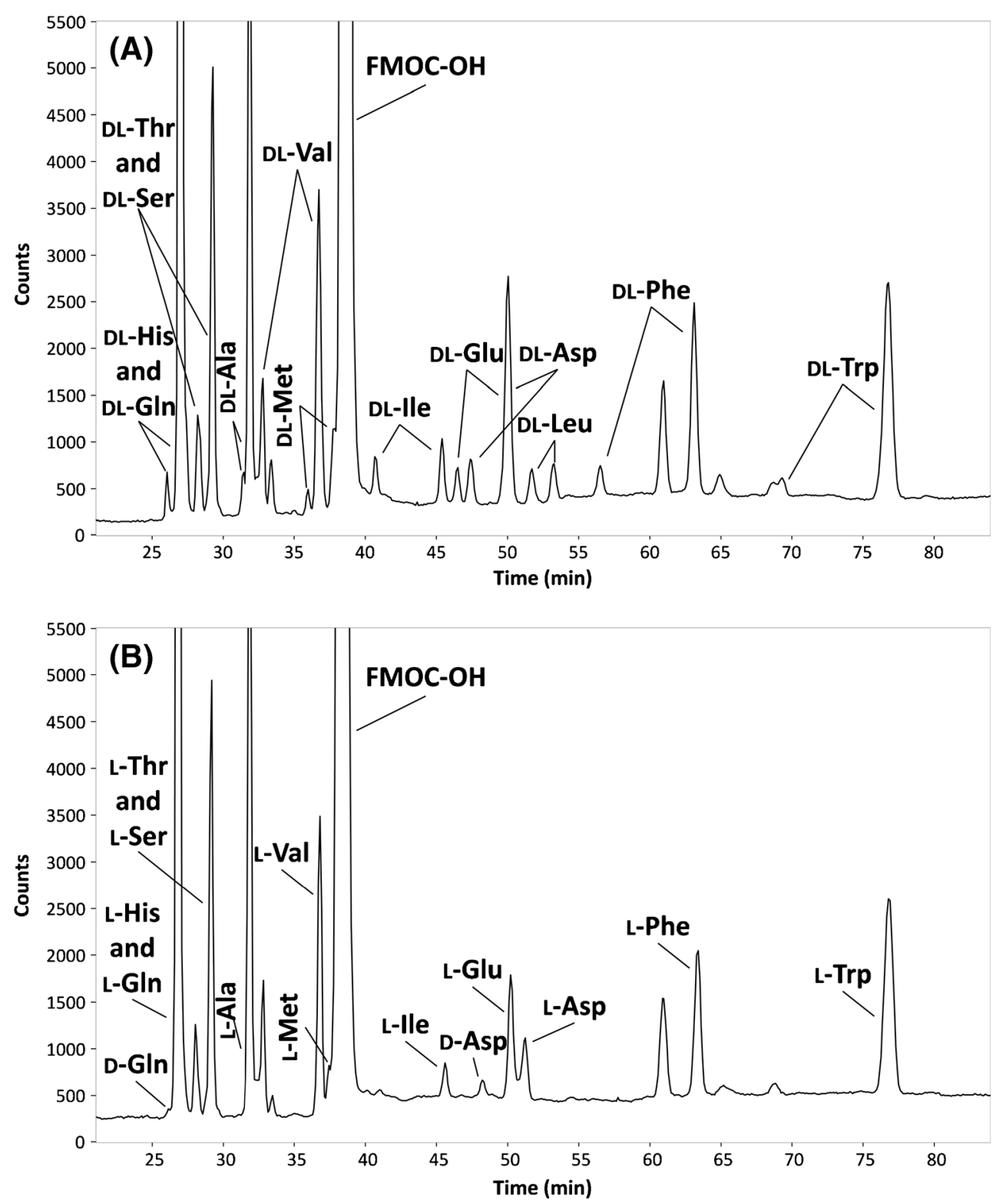

The developed CE-wrFlu method performance was compared to previously reported chiral CE-Flu methods for AAs, which almost all employ visible laser-induced excitation [16, 38-44] (Table 3). Using FMOC, the derivatization time ( $2 \mathrm{~min}$ ) was significantly shorter than for the earlier applied derivatization agents, in particular for FITC $(>12$ h), i.e., the most commonly used agent. The number of enantioseparated proteinogenic AAs (16 with chiral resolution >1.0) was also considerably higher than for previously reported methods (maximum of 8 AAs), while chiral separation of a mixture of 11 AAs could be achieved in a single run applying the currently presented method. Considering that our method employs regular lamp-based excitation, the achieved sensitivity is very satisfactory, exhibiting lower sample LODs than reported for most of the LIF-based chiral CE-Flu methods. The only chiral CEFlu method employing UV excitation published so far [41] showed more favorable sensitivity but was developed for one AA only (aspartic acid).

\section{Conclusion}

A new chiral CE method for AAs was developed encompassing fast derivatization with FMOC followed by selective separation employing a BGE with $\beta-C D$ and SDS and sensitive fluorescence detection. Efficient broad-band UV excitation of FMOC-AAs was achieved using a $\mathrm{Xe}-\mathrm{Hg}$ lamp in combination with a short-pass excitation filter. The optimized CE-Flu method enabled 


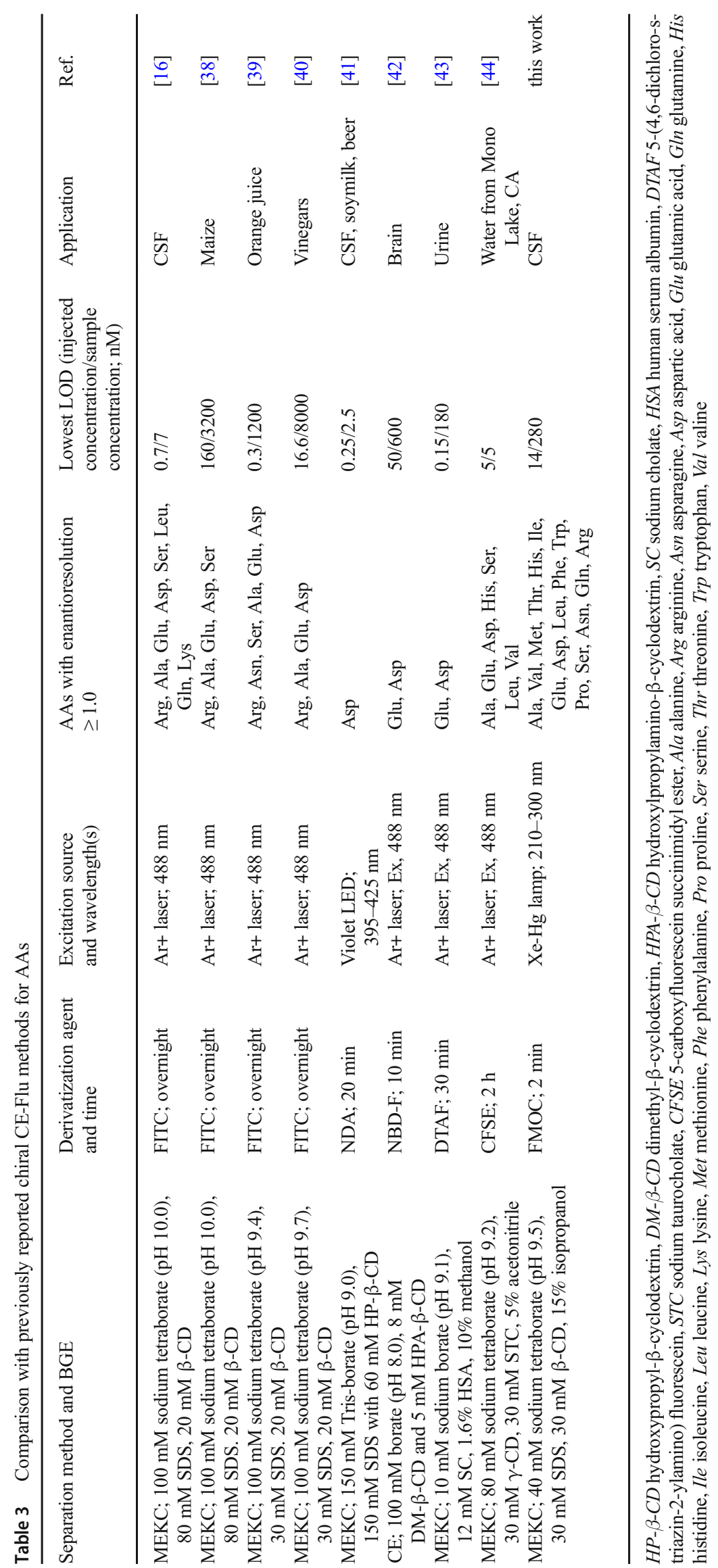


enantioseparation of 16 FMOC-DL-AAs with a resolution of 1.0 or higher. Use of wave-guiding principles for emission light collection provided good sensitivity, and further improvement of the $S / N$ for individual enantiomers was accomplished by wavelength-resolved CCD detection utilizing signal averaging along the wavelength axis. Resulting LODs for most AA enantiomers were between 10 and $100 \mathrm{nM}(2-15 \mathrm{ng} / \mathrm{mL}$ injected concentration), which is significantly better than the micromolar range LODs reported for similar analyses by chiral CE using UV absorbance detection [45-48]. The LODs for CE-Flu are similar to LODs obtained for FMOC-AAs using LIF detection employing a home-built UV laser [49]. Compared to chiral FMOC-AA analysis using CE-MS, LODs for CE-Flu were better with a factor of 3 for arginine and isoleucine up to a factor of 85 for valine in aqueous solution [58]. The CE-Flu LOD for D-leucine in CSF was 11.9 times lower than the LOD for this AA obtained with CE coupled with mass spectrometry (CEMS) [58]. The new chiral CE-Flu method was demonstrated to allow separation and detection of AA enantiomers in CSF, providing sufficient resolution and sensitivity to probe low levels of endogenous D-aspartic acid and D-glutamine next to excess L-AAs.

Compared with several previously reported chiral CE-Flu methods for AAs using LIF detection, the presented method has the advantage of fast derivatization time ( $2 \mathrm{~min}$ ), number of AAs enantioseparated (16 in total; 11 in one run), and favorable sensitivity while employing straightforward lamp excitation. As for most other chiral CE-Flu methods, the LODs for real samples were compromised by a dilution of the derivatized sample before injection, which was needed to avoid interferences by matrix components and overloading of the unreacted FMOC. For the presented method a 10times dilution was adequate, whereas other methods required much higher dilutions, deteriorating the ultimate LODs $[16$, 38-44]. The intrinsic sensitivity of the new CE-Flu system would allow D-AA detection down to the low-nanomolar range, but that would require an improved sample cleanup.

Acknowledgments This work was financially supported by the Netherlands Organization for Scientific Research (ECHO project 711.011.003).

\section{Compliance with ethical standards}

Conflict of interest The authors declare no conflict of interest.

Open Access This article is distributed under the terms of the Creative Commons Attribution 4.0 International License (http:// creativecommons.org/licenses/by/4.0/), which permits unrestricted use, distribution, and reproduction in any medium, provided you give appropriate credit to the original author(s) and the source, provide a link to the Creative Commons license, and indicate if changes were made.

\section{References}

1. Elango R, Ball RO, Pencharz PB. Amino acid requirements in humans: with a special emphasis on the metabolic availability of amino acids. Amino Acids. 2009;37:19-27.

2. Wu G. Amino acids: metabolism, functions, and nutrition. Amino Acids. 2009;37:1-17.

3. Bruckner H, Becker D, Lupke M. Chirality of amino acids of microorganisms used in food biotechnology. Chirality. 1993;5:38592.

4. Friedman M, Gumbmann MR, Masters PM. Protein-alkali reactions: chemistry, toxicology, and nutritional consequences. Adv Exp Med Biol. 1984;177:367-412.

5. Koval D, Jiraskova J, Strisovsky K, Konvalinka J, Kasicka V. Capillary electrophoresis method for determination of D-serine and its application for monitoring of serine racemase activity. Electrophoresis. 2006;27:2558-66.

6. Kim PM, Duan X, Huang AS, Liu CY, Ming GL, Song H, et al. Aspartate racemase, generating neuronal D-aspartate, regulates adult neurogenesis. P Natl Acad Sci USA. 2010;107:3175-9.

7. Errico F, Napolitano F, Nistico R, Centonze D, Usiello A. D-aspartate: an atypical amino acid with neuromodulatory activity in mammals. Rev Neurosci. 2009;20:429-40.

8. Hashimoto A, Nishikawa T, Oka T, Takahashi K. Endogenous Dserine in rat brain: N-methyl-D-aspartate receptor-related distribution and aging. J Neurochem. 1993;60:783-6.

9. Snyder SH, Kim PM. D-amino acids as putative neurotransmitters: focus on D-serine. Neurochem Res. 2000;25:553-60.

10. Yekkala R, Meers C, Van Schepdael A, Hoogmartens J, Lambrichts I, Willems G. Racemization of aspartic acid from human dentin in the estimation of chronological age. Forensic Sci Int. 2006;159(Suppl 1):S89-94.

11. Fisher G, Lorenzo N, Abe H, Fujita E, Frey WH, Emory C, et al. Free D- and L-amino acids in ventricular cerebrospinal fluid from Alzheimer and normal subjects. Amino Acids. 1998;15:263-9.

12. Fuchs SA, de Sain-van der Velden MG, de Barse MM, Roeleveld MW, Hendriks M, Dorland L, et al. Two mass-spectrometric techniques for quantifying serine enantiomers and glycine in cerebrospinal fluid: potential confounders and age-dependent ranges. Clin Chem. 2008;54:1443-50.

13. Luykx JJ, Bakker SC, van Boxmeer L, Vinkers CH, Smeenk HE, Visser WF, et al. D-amino acid aberrations in cerebrospinal fluid and plasma of smokers. Neuropsychopharmacology. 2013;38: 2019-26.

14. Fuchs SA, Berger R, Klomp LW, de Koning TJ. D-amino acids in the central nervous system in health and disease. Mol Genet Metab. 2005;85:168-80.

15. Bendikov I, Nadri C, Amar S, Panizzutti R, De Miranda J, Wolosker H, et al. A CSF and postmortem brain study of Dserine metabolic parameters in schizophrenia. Schizophr Res. 2007;90:41-51.

16. Samakashvili S, Ibanez C, Simo C, Gil-Bea FJ, Winblad B, Cedazo-Minguez A, et al. Analysis of chiral amino acids in cerebrospinal fluid samples linked to different stages of Alzheimer disease. Electrophoresis. 2011;32:2757-64.

17. Erny GL, Cifuentes A. Liquid separation techniques coupled with mass spectrometry for chiral analysis of pharmaceuticals compounds and their metabolites in biological fluids. J Pharmaceut Biomed. 2006;40:509-15.

18. Hernandez-Borges J, Rodriguez-Delgado MA, Garcia-Montelongo FJ, Cifuentes A. Chiral analysis of pollutants and their metabolites by capillary electromigration methods. Electrophoresis. 2005;26: 3799-813.

19. Simo C, Barbas C, Cifuentes A. Chiral electromigration methods in food analysis. Electrophoresis. 2003;24:2431-41. 
20. Chankvetadze B. Recent trends in enantioseparations using capillary electromigration techniques. Trac-Trend Anal Chem. 1999;18: 485-98.

21. Spanik I, Horvathova G, Janacova A, Krupcik J. On the use of solid phase ion exchangers for isolation of amino acids from liquid samples and their enantioselective gas chromatographic analysis. J Chromatogr A. 2007;1150:145-54

22. Chen $\mathrm{S}$. The enantioseparation of amino acids on a teicoplanin chiral stationary phase using non-aqueous mobile phases after pre-column derivatization with sulfur-containing reagents: the considerations of mobile phase composition and analyte structure variation on resolution enhancement. Biomed Chromatogr. 2006;20: 718-28.

23. Bhushan R, Nagar H. Indirect enantioseparation of proteinogenic amino acids using naproxen-based chiral derivatizing reagent and HPLC. Biomed Chromatogr. 2013;27:750-6.

24. Erbe T, Bruckner H. Chiral amino acid analysis of vinegars using gas chromatography - selected ion monitoring mass spectrometry. Z Lebensm Unters F A. 1998;207:400-9.

25. Erbe T, Bruckner H. Studies on the optical isomerization of dietary amino acids in vinegar and aqueous acetic acid. Eur Food Res Technol. 2000;211:6-12.

26. Blomberg LG, Wan H. Determination of enantiomeric excess by capillary electrophoresis. Electrophoresis. 2000;21:1940-52.

27. Guan J, Yan F, Shi S, Wang S. Optimization and validation of a new $\mathrm{CE}$ method for the determination of pantoprazole enantiomers. Electrophoresis. 2012;33:1631-6.

28. Tang W, Ong TT, Ng SC. Chiral separation of dansyl amino acids in capillary electrophoresis using mono-(3-methyl-imidazolium)-beta-cyclodextrin chloride as selector. J Sep Sci. 2007;30:1343-9.

29. Fradi I, Servais AC, Lamalle C, Kallel M, Abidi M, Crommen J, et al. Chemo- and enantio-selective method for the analysis of amino acids by capillary electrophoresis with in-capillary derivatization. $\mathrm{J}$ Chromatogr A. 2012;1267:121-6.

30. Sanchez-Hernandez L, Dominguez-Vega E, Montealegre C, Castro-Puyana M, Marina ML, Crego AL. Potential of vancomycin for the enantiomeric resolution of FMOC-amino acids by capillary electrophoresis-ion-trap-mass spectrometry. Electrophoresis. 2014;35:1244-50.

31. Wan H, Blomberg LG. Enantioseparation of amino acids and dipeptides using vancomycin as chiral selector in capillary electrophoresis. Electrophoresis. 1996;17:1938-44.

32. Verleysen K, Vandijck J, Schelfaut M, Sandra P. Enantiomeric separations in capillary electrophoresis using 18-crown-6tetracarboxylic acid $(18 \mathrm{C} 6 \mathrm{H}(4))$ as buffer additive. Hrc-J High Res Chrom. 1998;21:323-31.

33. Singh NS, Paul RK, Sichler M, Moaddel R, Bernier M, Wainer IW. Capillary electrophoresis-laser-induced fluorescence (CE-LIF) assay for measurement of intracellular D-serine and serine racemase activity. Anal Biochem. 2012;421:460-6.

34. Nouadje G, Rubie H, Chatelut E, Canal P, Nertz M, Puig P, et al. Child cerebrospinal fluid analysis by capillary electrophoresis and laser-induced fluorescence detection. J Chromatogr A. 1995;717: 293-8.

35. Siri N, Lacroix M, Garrigues JC, Poinsot V, Couderc F. HPLCfluorescence detection and MEKC-LIF detection for the study of amino acids and catecholamines labelled with naphthalene-2,3dicarboxyaldehyde. Electrophoresis. 2006;27:4446-55.

36. Taga A, Honda S. Derivatization at capillary inlet in highperformance capillary electrophoresis - its reliability in quantification. J Chromatogr A. 1996;742:243-50.

37. Simo C, Rizzi A, Barbas C, Cifuentes A. Chiral capillary electrophoresis-mass spectrometry of amino acids in foods. Electrophoresis. 2005;26:1432-41.
38. Herrero M, Ibanez E, Martin-Alvarez PJ, Cifuentes A. Analysis of chiral amino acids in conventional and transgenic maize. Anal Chem. 2007;79:5071-7.

39. Simo C, Barbas C, Cifuentes A. Sensitive micellar electrokinetic chromatography-laser-induced fluorescence method to analyze chiral amino acids in orange juices. J Agr Food Chem. 2002;50:5288-93.

40. Carlavilla D, Moreno-Arribas MV, Fanali S, Cifuentes A. Chiral MEKC-LIF of amino acids in foods: analysis of vinegars. Electrophoresis. 2006;27:2551-7.

41. Lin KC, Hsieh MM, Chang CW, Lin EP, Wu TH. Stacking and separation of aspartic acid enantiomers under discontinuous system by capillary electrophoresis with light-emitting diode-induced fluorescence detection. Talanta. 2010;82:1912-8.

42. Wagner Z, Tabi T, Jako T, Zachar G, Csillag A, Szoko E. Chiral separation and determination of excitatory amino acids in brain samples by CE-LIF using dual cyclodextrin system. Anal Bioanal Chem. 2012;404:2363-8.

43. Wang S, Fan L, Cui S. CE-LIF chiral separation of aspartic acid and glutamic acid enantiomers using human serum albumin and sodium cholate as dual selectors. J Sep Sci. 2009;32:3184-90.

44. Creamer JS, Mora MF, Willis PA. Enhanced resolution of chiral amino acids with capillary electrophoresis for biosignature detection in extraterrestrial samples. Anal Chem. 2017;89:1329-37.

45. Han Y, Chen Y. On-column labeling technique and chiral CE of amino acids with mixed chiral selectors and UV detection. Electrophoresis. 2007;28:2765-70.

46. Chan KC, Muschik GM, Issaq HJ. Enantiomeric separation of amino acids using micellar electrokinetic chromatography after precolumn derivatization with the chiral reagent 1-(9-fluorenyl)-ethyl chloroformate. Electrophoresis. 1995;16:504-9.

47. Wan H, Andersson PE, Engström A, Blomberg LG. Direct and indirect chiral separation of amino acids by capillary electrophoresis. J Chromatogr A. 1995;704:179-93.

48. Wan H, Blomberg LG. Optimized chiral separation of 20 amino acids derivatized with 9-fluorenylmethyl chloroformate using cyclodextrins as chiral selectors in capillary electrophoresis. J Chromatogr Sci. 1996;34:540-6.

49. Chan KC, Janini GM, Muschik GM, Issaq HJ. Laser-induced fluorescence detection of 9-fluorenylmethyl chloroformate derivatized amino acids in capillary electrophoresis. J Chromatogr A. 1993;653:93-7.

50. Caslavska J, Thormann W. Monitoring of drugs and metabolites in body fluids by capillary electrophoresis with $\mathrm{XeHg}$ lamp-based and laser-induced fluorescence detection. Electrophoresis. 2004;25: 1623-31.

51. Priego Capote F, Rodriguez JM, Luque de Castro MD. Determination of phenolic compounds in grape skin by capillary electrophoresis with simultaneous dual fluorescence and diode array absorption detection after dynamic superheated liquid leaching. J Chromatogr A. 2007;1139:301-7.

52. Priego-Capote F, Luque de Castro MD. Determination of B2 and B6 vitamers in serum by capillary electrophoresis-molecular fluorescence-charge coupled detector. Electrophoresis. 2005;26:2376-83.

53. Pobozy E, Michalski A, Sotowska-Brochocka J, Trojanowicz M. Determination of melatonin and its precursors and metabolites using capillary electrophoresis with UV and fluorometric detection. J Sep Sci. 2005;28:2165-72.

54. Mallampati S, Van Aerschot A, Hoogmartens J, Van Schepdael A. Analysis of dideoxyadenosine triphosphate by CE with fluorescence detection. I. Derivatization through the phosphate group. Electrophoresis. 2007;28:3948-56.

55. Radenovic DC, de Kort BJ, Somsen GW. Lamp-based native fluorescence detection of proteins in capillary electrophoresis. $\mathrm{J}$ Chromatogr A. 2009;1216:4629-32. 
56. de Kort BJ, ten Kate GA, de Jong GJ, Somsen GW. Capillary electrophoresis with lamp-based wavelength-resolved fluorescence detection for the probing of protein conformational changes. Anal Chem. 2011;83:6060-7.

57. de Kort BJ, de Jong GJ, Somsen GW. Lamp-based wavelengthresolved fluorescence detection for protein capillary electrophoresis: setup and detector performance. Electrophoresis. 2010;31: 2861-8.

58. Prior A, Sanchez-Hernandez L, Sastre-Torano J, Marina ML, de Jong GJ, Somsen GW. Enantioselective analysis of proteinogenic amino acids in cerebrospinal fluid by capillary electrophoresis-mass spectrometry. Electrophoresis. 2016;37:2410-9.

59. Gustavsson B, Betnér I. Fully automated amino acid analysis for protein and peptide hydrolysates by precolumn derivatization with 9-fluorenyl methylchloroformate and 1-aminoadamantane. J Chromatogr A. 1990;507:67-77.

60. Thorsen G, Bergquist J. Chiral separation of amino acids in biological fluids by micellar electrokinetic chromatography with laser-induced fluorescence detection. J Chromatogr B. 2000;745:389-97.

61. Prior A, Moldovan RC, Crommen J, Servais AC, Fillet M, de Jong GJ, et al. Enantioselective capillary electrophoresis-mass spectrometry of amino acids in cerebrospinal fluid using a chiral derivatizing agent and volatile surfactant. Anal Chim Acta. 2016;940:150-8.

62. Fisher GH, Petrucelli L, Gardner C, Emory C, Frey WH, Amaducci $\mathrm{L}$, et al. Free D-amino acids in human cerebrospinal fluid of Alzheimer disease, multiple sclerosis and healthy control subjects. Mol Chem Neuropathol. 1994;23:115-24. 